\title{
Vascularized Proximal Fibular Epiphyseal Transfer for Shoulder Reconstruction after Tumor Resection: A Case Report
}

\author{
Emmanuel P. Estrella, 1,2 Ellen Y. Lee ${ }^{1,2}$ and Edward HM Wang 1,2 \\ ${ }^{1}$ Microsurgery Unit, Department of Orthopedics, College of Medicine and Philippine General Hospital, University of the Philippines Manila \\ ${ }^{2}$ ASTRO (Advanced Study and Research in Orthopedics) \\ Study Group, National Institutes of Health, University of the Philippines Manila
}

\begin{abstract}
We present a case of an 8-year-old girl with a high grade osteogenic sarcoma of the proximal humerus treated with wide resection and vascularized proximal fibular epiphyseal transfer. At 5 years after reconstruction, the patient is tumor free and had a Musculoskeletal Tumor Score of $26 / 30$ or $86.7 \%$. The functional outcomes in terms of shoulder range of motion and pain were good. Complications include transient peroneal nerve palsy and mild valgus instability of the knee.
\end{abstract}

Key Words: fibula transfer, epiphyseal transfer, shoulder reconstruction, osteosarcoma

\section{Introduction}

The use of vascularized fibula graft has been one of the most common procedures employed in the reconstruction of long bone defects as a result of trauma, tumor resection, infection or congenital abnormalities of both the upper and lower extremities., ${ }^{1,2}$ The advantages of vascularized fibular grafting have been well described in the literature. The procedure provides rapid union, ${ }^{3}$ is ideal for defects greater than five centimeters, ${ }^{4}$ and has the ability to tolerate physiologic loading after fixation..$^{5}$ Aside from the biological stability and union that the vascularized fibula offers, another advantage is the restoration of joint motion and bone growth potential. This is especially true in children where resected tumors lie in areas such as the distal radius and proximal humerus where conventional treatments such as prosthesis replacement and allograft implantation are difficult to achieve. ${ }^{6,7}$ The microvascular transfer of the proximal part of the fibula, including the open physis, offers the potential advantage of preserving joint motion and the potential for longitudinal growth. ${ }^{8}$ Previous reports on microvascular epiphyseal transfer of the fibula for proximal humeral reconstruction have been reported with good results. ${ }^{9,10}$

Corresponding author: Emmanuel P. Estrella, MD

Microsurgery Unit, Department of Orthopedics

Philippine General Hospital

University of the Philippines Manila

Taft Avenue, Ermita, Manila 1000 Philippines

Telephone: +6325548466

Email: estee96@yahoo.com
We report a case of an 8-year-old female, diagnosed with osteosarcoma of the proximal humerus where after wide resection, the bone defect was treated by vascularized fibular epiphyseal transfer to maintain shoulder motion.

\section{Case Report}

An 8-year-old, right-handed girl was referred for pain and limitation of shoulder motion on the right shoulder of 3.5 months duration. There was no history of trauma and the range of motion from the elbow to the hand was normal. Radiographic findings revealed a large mass on the proximal humerus (Figure 1). The initial diagnosis was a bone tumor. Additional diagnostic examinations included a full body bone scan and a CT scan of the chest, which were negative for any lesions. An open biopsy was performed for histologic diagnosis. Histology provided a diagnosis of high grade osteosarcoma. The patient was then advised neoadjuvant chemotherapy.

After preoperative chemotherapy, the patient underwent wide excision of the mass. The patient was positioned semi-supine with a sandbag under the right shoulder and the right hip. The fibula graft on the same side was harvested at the same time as the tumor excision. Almost all of the deltoid muscle was removed. Part of the insertions of the rotator cuff muscles and the origins of the conjoint tendons of the coracobrachialis and biceps muscles were taken with the tumor. All nerves were preserved. The final osseous defect was $15 \mathrm{~cm}$.

Surgical Technique of Vascularized Proximal Fibula Epiphyseal transfer

A long incision on the lateral leg, slightly anterior to the fibula was planned, extending a few centimeters proximal to the fibula head (Figure 2). We isolated the anterior tibial artery and vein, which was shown to have a dominant blood supply to the proximal fibular epiphysis via epiphyseal branches $^{11}$ (Figure 3). We used a tourniquet to facilitate the fibula harvest. We started our dissection by identifying the peroneal nerve between the tibialis anterior and extensor digitorum longus and made our way proximally to the fibula neck. The peroneal nerve and several small branches of the nerve were then separated from the anterior tibial artery under intraoperative nerve stimulation guidance. The 
nerves were incised if they could not be separated from the artery and repaired if they innervate a significant part of the peroneal muscles. The anterior tibial artery was then traced distally up to the level of fibula resection, which was 14-15 centimeters. The distal dissection of the diaphyseal fibula was harvested like the usual fibula transfer. During the harvest, we incised the interosseous membrane longitudinally to free the fibula, including longitudinal strips of muscle in order to protect the small branches to the fibular periosteum.

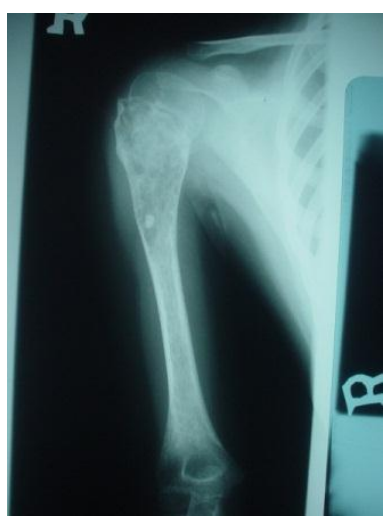

Figure 1. Radiograph showing a large, osteoblastic-osteolytic lesion on the proximal humerus.

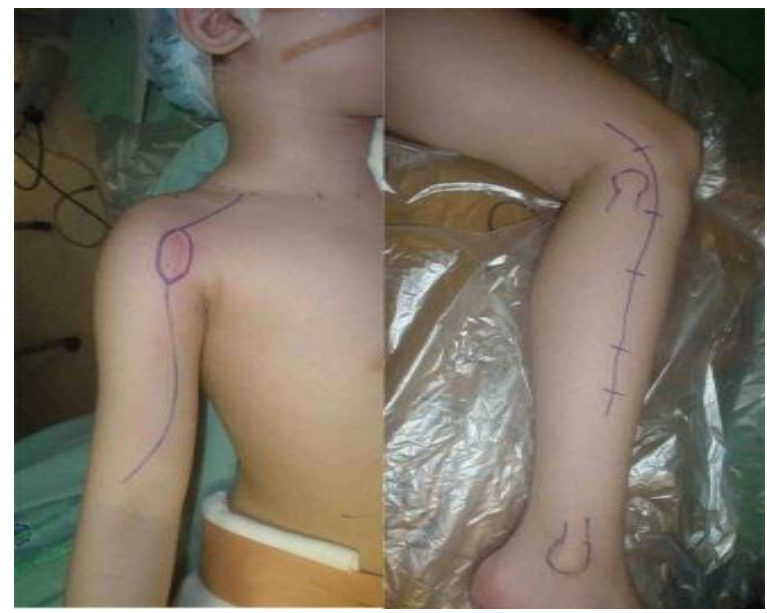

Figure 2. An incision for the wide resection of the osteosarcoma of the proximal humerus with inclusion of the biopsy site (left). The antero-lateral approach to the proximal fibular epiphysis was employed. The incision was just anterior to the fibula extending to the lateral collateral ligament of the knee (right).

In the area of the fibular neck, the peroneus longus and extensor digitorum longus were transected transversely to expose the common peroneal nerve. Once the proximal tibiofibular joint was opened, the biceps tendon was divided longitudinally and the posterior half was left with the fibula.
The anterior half was used to reinforce the lateral collateral ligament that was detached from the fibular head and attached to lateral tibial metaphysis through the periosteum with non-absorbable sutures and tested for stability (Figure 4). The anterior tibial artery and its venae comitantes were then isolated and ligated proximally and distally. Prior to ligation, the anterior tibial artery was clamped for 30 minutes and the tourniquet was released to observe for the vascularity of the leg. Once the vascularity was adequate, the anterior tibial artery was ligated and the peroneal vessels were carefully separated from the fibula. When the fibula was free, it was then harvested for transfer.

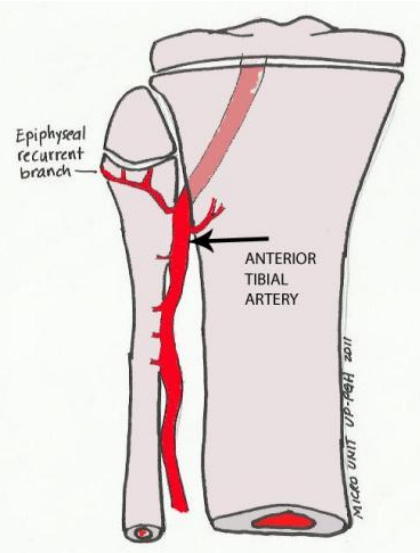

Figure 3. The epiphyseal arteries supplying the proximal fibular epiphysis come from the anterior tibial artery. The anterior tibial artery also supplies the proximal two-thirds of the fibular shaft. Broken line arrow shows area where the anterior tibial artery was ligated.

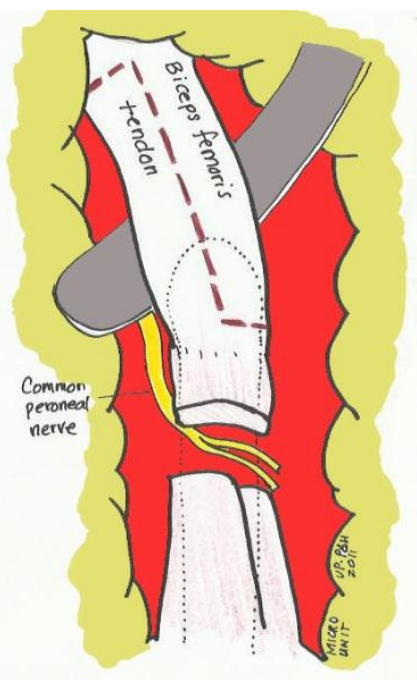

Figure 4. Once isolated, the biceps femoris was split longitudinally. The posterior half was left with the fibular epiphysis which served to stabilize the fibula to the glenoid. The anterior half was reattached to the proximal tibia for reconstruction of the lateral collateral ligament. 
Once transferred to the defect at the proximal humerus, a six-hole mini-DCP (dynamic compression plate) was used to stabilize the distal fragment and the proximal head was fixed to the glenoid with a smooth $0.062 \mathrm{k}$-wire (Figure 5). A reverse flow arterial model was used as reported previously ${ }^{12}$ to supply the fibula using the distal anterior tibial artery anastomosed to a branch of the brachial artery using 10-0 nylon suture under the operating microscope. The external diameters of the vessel were as follows: the anterior tibial artery was $1.0 \mathrm{~mm}$ and the recipient artery on the arm (radial recurrent artery) was $0.7 \mathrm{~mm}$. We then observed which vein was the dominant one and anastomosed it to the cephalic vein (external diameter: $2 \mathrm{~mm}$ ), which was preserved during tumor resection. The remaining half of the biceps tendon on the fibular head was weaved around the remaining glenoid labrum and capsule to hold the fibula. The supraspinatus tendon was attached to the posterior part of the fibula head and the latissimus dorsi tendon was attached to the anterior part of the fibula to preserve external and internal rotation of the shoulder (Figure 6). Once adequate arterial and venous flow was observed from the fibula and vessel anastomosis, routine hemostasis and closure with negative suction drains were performed. Tourniquet time was 118 minutes and the total operative time was 12 hours. Blood loss was $400 \mathrm{cc}$ and the patient was transfused one unit of packed RBC postoperatively.

Postoperatively, the knee was placed in an immobilizer for 6-8 weeks non-weight bearing and the upper extremity was placed on a sling with a pin from the fibular head to the glenoid fossa.

A foot drop was noted postoperatively and a splint was fabricated. Recovery was noted at 2 months post-op and normal ankle dorsi flexion was evident at 6 months post-op. Pin was removed 6 weeks postoperatively and gradual range of motion exercises were encouraged at the shoulder. Distal bone union was observed at 2 months post-op.

A bone scan was performed three months after the procedure to assess vascularity of the epiphysis. This was repeated at 12 months postoperatively, showing continued uptake of the transplanted fibular epiphysis (Figure 7).

At 3 weeks post-op, the patient had postoperative chemotherapy. On histopathology, the tumor had clear margins and a necrosis rate of $<90 \%$.

At 5 years follow-up, the patient remained disease-free. There was mild varus knee instability but the patient had no limitations in walking and running. The Enneking musculoskeletal score ${ }^{13}$ was $26 / 30$ or $86.67 \%$.

The active lateral abduction of the shoulder was $0-40^{\circ}$, forward flexion was $0-60^{\circ}$, internal rotation was $0-90^{\circ}$, and external rotation was $0-45^{\circ}$ (from the neutral position). There was no pain and the patient could use her hand and upper extremity for activities of daily living like dressing, eating and holding light objects.
Clinically, the reconstructed extremity was shorter by 4 centimeters. On radiographs, remodeling of the fibular head was evident, but minimal hypertrophy was observed. The length of the fibula was compared to the initial radiographs taken at the same institution. The fibula had lengthened approximately $0.36 \mathrm{~cm} /$ year (Figure 8).

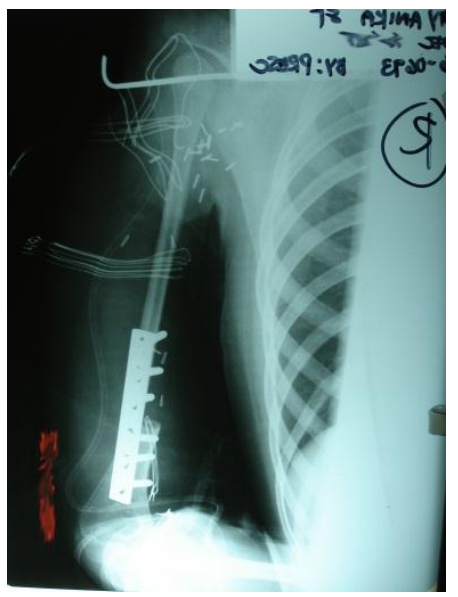

Figure 5. The fibula transfer was also transfixed with a single $0.062 \mathrm{k}$-wire to the glenoid, aside from using part of the biceps femoris as an additional support to the shoulder reconstruction. The pin was maintained for 6 weeks.

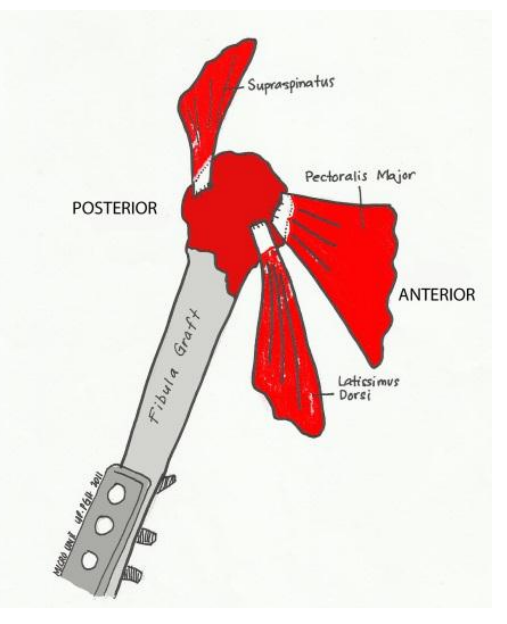

Figure 6. Shoulder reconstruction was done by attaching the supraspinatus tendon posteriorly, and the pectoralis major and latissimus dorsi muscles anteriorly to the fibular head to restore external rotation/abduction and internal rotation adduction, respectively.

\section{Discussion}

Vascularized epiphyseal transfer of the fibula was chosen to fulfill three problems after proximal humeral resection: replacement of lost bone, preservation of shoulder motion, and maintenance of growth. To achieve these results, vascularized proximal fibular epiphyseal transfer is the procedure of choice. In 2007, Innocenti and colleagues 
reported 17 cases of vascularized epiphyseal transplant for proximal humeral reconstruction. ${ }^{14}$ Five of the 17 reconstructions had early physeal closure. This was attributed to the use of the peroneal artery pedicle in two cases, and damage to the anterior tibial artery during dissection in three cases. The average annual growth of their transfers was $0.7-1.35 \mathrm{~cm}$. The vascularized epiphyseal transfer in this case report was based on the anterior tibial artery. Shoulder reconstruction was achieved with this procedure. Our results showed that shoulder external and internal rotation was very good: $45^{\circ}$ and $90^{\circ}$, respectively. However, lateral abduction was only $40^{\circ}$ and forward flexion was only $60^{\circ}$. The inclusion of most of the deltoid in the wide excision prevented the reconstruction from achieving a greater degree of lateral abduction. Additional procedures such as trapezius transfers for lateral abduction may be used, as applied in deltoid paralysis and brachial plexus injuries. ${ }^{15}$ Also, as reported by other authors, ${ }^{7,9}$ remodeling of the fibular head was evident in this case. This is another advantage of using a vascularized epiphyseal transfer in joint reconstruction. However, the growth and remodelling of the vascularized epiphyseal transfer may be affected by the patient's age, mechanical and biologic factors at the recipient site, the quality of the blood supply, and the use of postoperative chemotherapy. ${ }^{16}$ In terms of graft hypertrophy, measured using the graft hypertrophy index of DeBoer and Wood, ${ }^{17}$ the graft hypertrophied by $25 \%$.

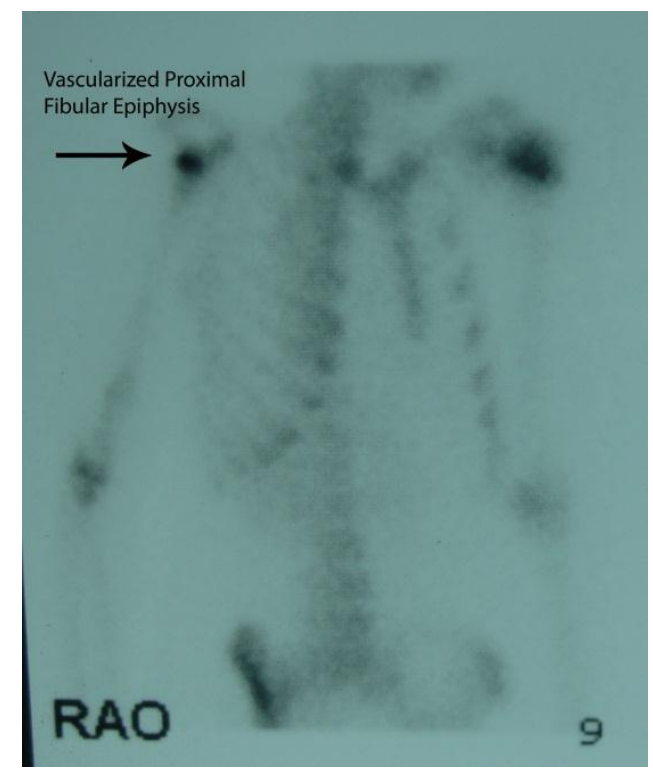

Figure 7. Total body bone scan at 1 year postoperatively showing continued uptake on the vascularized proximal fibular epiphysis reconstruction of the right shoulder (black arrow). This demonstrates continued vascularity and viability of the proximal fibular epiphysis.

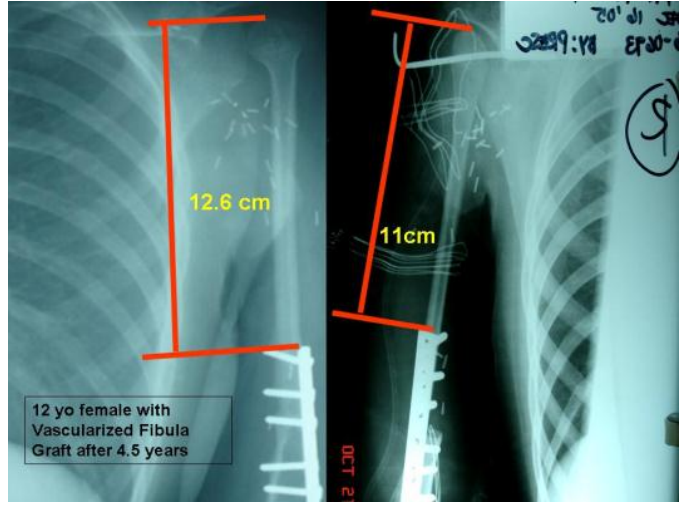

Figure 8. Mirror radiographs taken immediately after surgery (left) and approximately 54 months after reconstruction (right). Remodelling of the fibular head was evident by change in the shape of the fibular head and lengthening of the graft by $1.6 \mathrm{~cm}$ in 54 months or approximately $0.36 \mathrm{~cm} /$ year. Graft hypertrophy was $25 \%$.

Other options for shoulder reconstruction include shoulder arthrodesis, ${ }^{18}$ which have been reported to have an average MSTS score of 22 points (73.33\%) and a complication rate of $43 \%$.

\section{Summary}

A variety of options exists in the reconstruction of the proximal humerus after tumor resection. However, the choice of the reconstructive procedure will ultimately depend on the needs of the patient and the surgical expertise of the surgeon. The goal for this patient was adequate tumor resection, osteo-articular reconstruction that can provide some degree of shoulder motion and longitudinal growth. This was achieved with a $15-\mathrm{cm}$ vascular proximal epiphyseal transfer based on the anterior tibial artery. At 5 years post-reconstruction, the patient remains tumor free and has good function of the shoulder and upper extremity.

\section{References}

1. Heitman C, Levin LS. Applications of the vascularized fibula for upper extremity reconstruction. Tech Hand Up Extrem Surg. 2003; 7(1):12-7.

2. Heller L, Levin LS. Lower extremity microsurgical reconstruction. Plast Reconstr Surg. 2001; 108(4):1029-41.

3. Doi K, Tominaga S, Shibata T. Bone grafts with microvascular anastomoses of vascular pedicles: an experimental study in dogs. J Bone Joint Surg Am. 1977; 59(6):809-15.

4. Dell PC, Burchardt H, Glowczewskie FP Jr. A roentgenographic, biomechanical, and histological evaluation of vascularized and nonvascularized segmental fibular canine autografts. J Bone Joint Surg Am. 1985; 67(1):105-12.

5. Moore JB, Mazur JM, Zehr D, Davis PK, Zook EG. A biomechanical comparison of vascularized and conventional autogenous bone grafts. Plast Reconstr Surg. 1984; 73(3):382-6. 
6. Innocenti M, Ceruso M, Manfrini M, et al. Free vascularised growthplate transfer after bone tumor resection in children. J Reconstr Microsurg. 1998; 14(2):137-43.

7. Innocenti M, Ceruso M, Delcroix L. Vascularised epiphyseal transfer in upper limb skeletal reconstruction in children. Indications and operative technique. In: Schuind F, de Fontaine S, Van Geertrayden J, Soucacos PN, editors. Advances in upper and lower extremity microvascular reconstructions. Singapore: World Scientific; 2002. pp. 90-105.

8. Nunley JA, Spiegl PV, Goldner RD, Urbaniak JR. Longitudinal epiphyseal growth after replantation and transplantation in children. J Hand Surg Am. 1987; 12(2):274-9.

9. Shea KG, Coleman SS, Coleman DA. Growth of the proximal fibula physis and remodelling of the epiphysis after microvascular transfer: a case report. J Bone Joint Surg Am. 1997; 79(4):583-6.

10. Onoda S, Sakuraba M, Asano T, et al. Use of vascularized free fibular head grafts for upper limb oncologic reconstruction. Plast Reconstr Surg. 2011; 127(3):1244-53.

11. Taylor GI, Wilson KR, Rees MD, Corlett RJ, Cole WG. The anterior tibial vessels and their role in epiphyseal and diaphyseal transfer of the fibula: experimental study and clinical applications. Br J Plast Surg. 1988; 41(5):451-69.
12. del Pinal F, Taylor GI. The deep venous system and reverse flow flaps. Br J Plast Surg. 1993; 46(8):652-64.

13. Enneking WF, Dunham W, Gebhardt MC, Malawar M, Pritchard DJ. A system for the functional evaluation of reconstructive procedures after surgical treatment of tumors of the musculoskeletal system. Clin Orthop Relat Res. 1993; (286):241-6.

14. Innocenti M, Delcroix L, Romano GF, Capanna R. Vascularized epiphyseal transplant. Orthop Clin North Am. 2007; 38(1):95-101.

15. Rühmann O, Wirth CJ, Gossé F, Schmolke S. Trapezius transfer after brachial plexus palsy. Indications, difficulties and complications. J Bone Joint Surg Br. 1998; 80(1):109-13.

16. del Pinal F, Innocenti M. Evolving concepts in the management of the bone gap in the upper limb. Long and small defects. J Plast Reconstr Aesthet Surg. 2007; 60(7):776-92.

17. de Boer $\mathrm{HH}$, Wood MB. Bone changes in the vascularised fibular graft. J Bone Joint Surg Br. 1989; 71(3):374-8.

18. Fuchs B, O'Connor MI, Padgett DJ, Kaufman KR, Sim FH. Arthrodesis of the shoulder after tumor resection. Clin Orthop Relat Res. 2005; (436):202-7.
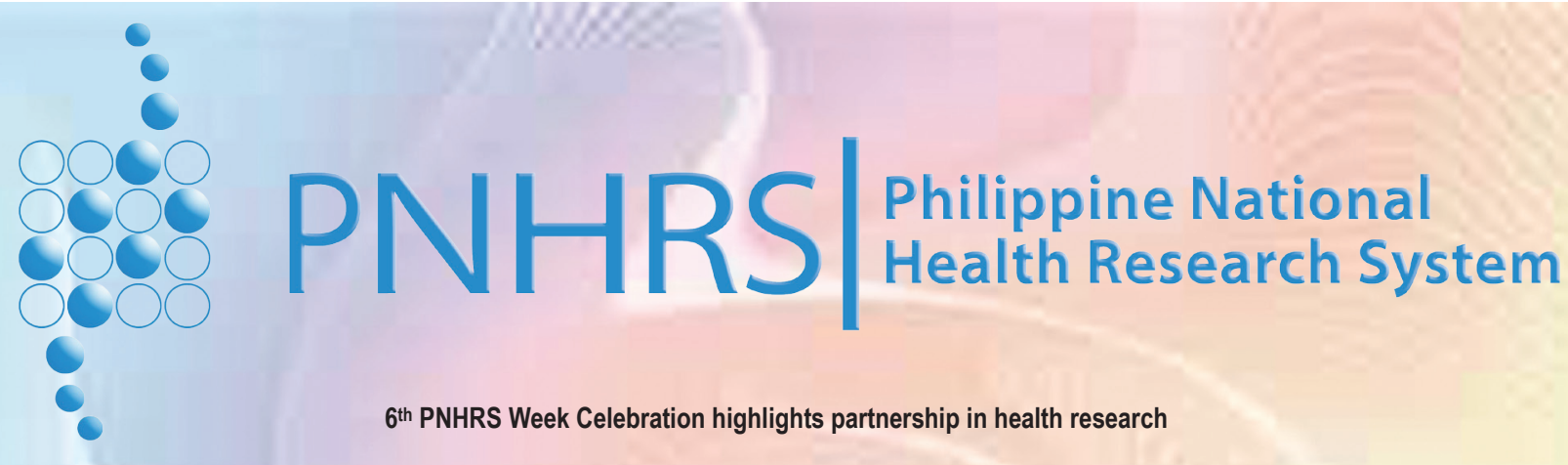

Health research stakeholders will gather again for the $6^{\text {th }}$ Philippine National Health Research System (PNHRS) Week celebration on August 8 - 10 , 2012 to be held at the Hotel Sofitel Philippine Plaza Manila. With the theme, "Sustaining Research Partnerships for Better Health," the event emphasizes the healthy cooperation of research stakeholders to generate new knowledge and innovations in support of the country's Kalusugang Pangkalahatan agenda.

This year's celebration will bring in the consortia chairs and committee chairs to parallel sessions on R\&D agenda, capacity building, research utilization, ethics and governance. As well, there will be plenary and concurrent sessions on topic relevant to strengthening health research collaboration.

More than 600 participants from all the 17 regional health research consortia are expected to participate in the event. For more information, please contact PNHRS secretariat at telephone numbers: (02) 8377534 or (02) 8377537 or visit PNHRS website at http://www.healthresearch.ph/.

PNHRS Week is held annually on the second week of August by virtue of Proclamation No. 1309 signed in 2007. 